\title{
Indentical synchronization in complete networks of reaction-diffusion equations of Fitzhugh-nagumo
}

\author{
Phan Van Long $\mathrm{Em}^{1^{*}}$ \\ ${ }^{1}$ An Giang University, Vietnam \\ *Corresponding author: pvlem@agu.edu.vn
}

\begin{tabular}{ll}
\hline \multicolumn{1}{c}{ ARTICLE INFO } & \multicolumn{1}{c}{ ABSTRACT } \\
\hline $\begin{array}{l}\text { DOI:10.46223/HCMCOUJS. } \\
\text { tech.en.8.2.346.2018 }\end{array}$ & \multicolumn{1}{c}{$\begin{array}{c}\text { Synchronization is a ubiquitous feature in many natural } \\
\text { systems and nonlinear science. This paper studies the } \\
\text { synchronization incomplete network consisting of } n \text { nodes. Each } \\
\text { node is connected to all other nodes by linear coupling and } \\
\text { represented by a reaction-diffusion system of FitzHugh-Nagumo }\end{array}$} \\
$\begin{array}{l}\text { Received: February 6 } 6^{\text {th }}, 2017 \\
\text { Revised: September 20 } 0^{\text {th }}, 2017 \\
\text { type which can be obtained by simplifying the famous Hodgkin- } \\
\text { Accepted: May 14th } 2018\end{array}$ & $\begin{array}{l}\text { Huxley model. From this complete network, the author seeks a } \\
\text { sufficient condition on the coupling strength to achieve } \\
\text { synchronization. The result shows that the more easily the nodes } \\
\text { synchronize, the bigger the degrees of the networks. Based on this }\end{array}$ \\
$\begin{array}{l}\text { Keywords: } \\
\text { complete network, coupling } \\
\text { strength, fitzhugh-nagumo } \\
\text { model, synchronization }\end{array}$ & $\begin{array}{l}\text { consequence, the author will test the theoretical result numerically } \\
\text { to see if there is a compromise. }\end{array}$ \\
\hline
\end{tabular}

\section{Introduction}

The FitzHugh-Nagumo model was introduced as a dimensional reduction of the wellknown Hodgkin-Huxley model (see, e.g., Ermentrout \& Terman, 2009; Hodgkin \& Huxley, 1952; Izhikevich, 2007; Keener \& Sneyd, 2009; Murray, 2010; Nagumo, Arimoto, \& Yoshizawa, 1962). It is more analytically tractable and maintains some biophysical meaning. The model has constituted a common form of two equations in the two variables $u$ and $v$. The first variable is the fast one called excitatory which represents the transmembrane voltage. The second is the slow recovery variable which describes the time dependence of several physical quantities, such as the electrical conductivity of ion currents across the membrane. The FitzHugh-Nagumo equations (FHN), using the notation in Ambrosio and Aziz-Alaoui (2012); Ambrosio (2009); Ambrosio and Aziz-Alaoui (2013), are given by,

$$
\left\{\begin{array}{l}
\varepsilon \frac{d u}{d t}=f(u)-v \\
\frac{d v}{d t}=a u-b v+c
\end{array}\right.
$$

where $a, b$ and $c$ are constants ( $a$ and $b$ are strictly positive), $0<\varepsilon \ll 1$ and $f(u)=-u^{3}+3 u$. 


$$
\left\{\begin{array}{l}
\varepsilon \frac{d u}{d t}=\varepsilon u_{t}=f(u)-v+d_{13} \Delta u \\
\frac{d v}{d t}=v_{t}=a u-b v+c
\end{array}\right.
$$

where $u=u(x, t), v=v(x, t),(x, t) \in \Omega \times \mathbb{R}^{+}, d_{u}$ is a positive constant, $\Delta u$ is the Laplace operator of $u, \Omega \subset \mathbb{R}^{n}$ is a regular bounded open set and with Neumann zero flux boundary conditions. This system allows the emergence of a variety of patterns and relevant phenomena in physiology (see, e.g., Ambrosio \& Aziz-Alaoui, 2012; Ambrosio \& Aziz-Alaoui, 2013). It is a system of two nonlinear partial differential equations of the incomplete parabolic type which describes the action potential and the recovery variable in the whole set of neurons. Note that the first equation is similar to the so-called cable equation, which describes the distribution of the potential along the axon of a single neuron (see, e.g., Ermentrout \& Terman, 2009; Izhikevich, 2005). For example, in figure 1, we have two solutions of system (1) corresponding to different values of $t$ on space $\Omega=[0 ; 100] \times[0 ; 100]$. Figure (a) represents, for $t=0$, the iso values $u\left(x_{1}, x_{2}, 0\right)$ of one solution of system (1). Figure (b) represents, for $t=190$, the iso values $u\left(x_{1}, x_{2}, 190\right)$ of one solution of system (1). Such a solution is called spiral one obtained by a particular choice of initial conditions.

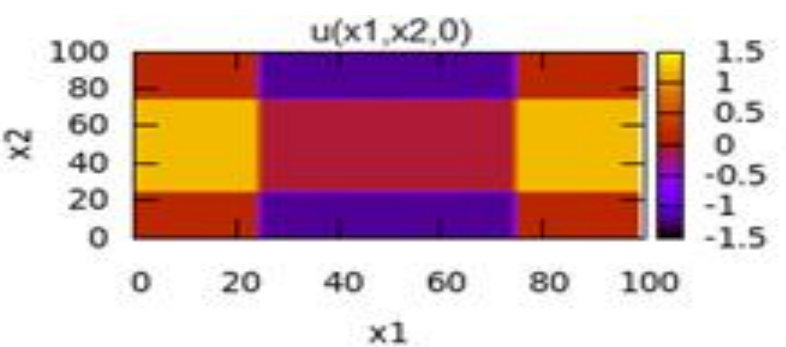

(a)

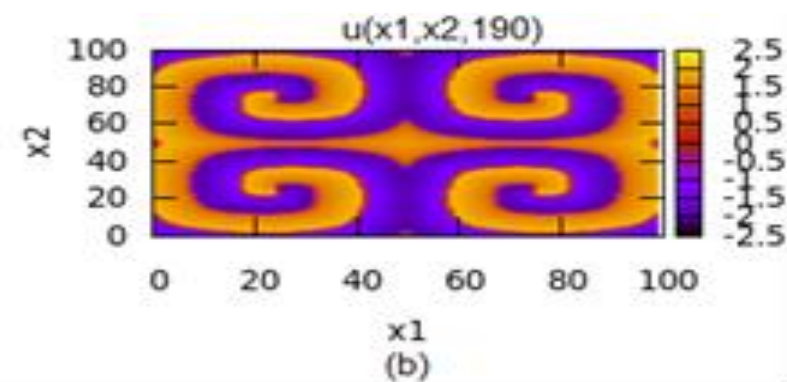

(b)

Figure 1. (a) represents, for $\mathrm{t}=0$, the iso values $u(x 1, x 2,0)$ of one solution of system (1). (b) represents, for $\mathrm{t}=190$, the iso values $u\left(x_{1}, x_{2}, 190\right)$ of one solution of system (1). Such a solution is called spiral one obtained by a particular choice of initial conditions

After having the model of a neuron, we consider a network of n coupled systems (1) based on FHN type as follows:

$$
\left\{\begin{array}{l}
\varepsilon u_{i t}=f\left(u_{i}\right)-v_{i}+d_{1 u_{i}} \Delta u_{u_{i}}-h\left(u_{i}, v_{i}\right) \\
v_{i t}=a u_{i}-b v_{i}+c
\end{array} \quad i, j=1, \ldots, n, i \neq j,\right.
$$

where $\left(u_{i}, v_{i}\right), i=1,2, \ldots, n$ is defined by $(1)$.

The function $h$ is the coupling function that determines the type of connection between neurons $i$ and $j$. Connections between neurons are essential of two types: a chemical which is 
much more abundant, and electrical. If the connections are made by electrical synapse, the coupling is linear and given by the function:

The parameter $h\left(u_{i}, v_{i}\right)=g_{g n} \sum_{j=1}^{n} c_{i j}\left(u_{i}-u_{j}\right), \quad i=1,2, \ldots, n$.

The parameter $g_{\text {syn }}$ represents the coupling strength. The coefficients $c_{i j}$ are the elements of the connectivity matrix $C_{n}=$ $\left(c_{i j}\right)_{n \times n}$, defined by:

$$
\left\{\begin{array}{l}
c_{i j}=1 \text { if } i \text { and } j \text { arecoupled } \\
c_{i j}=0 \text { if } i \text { and } j \text { arecoupled }
\end{array} \quad i, j=1,2, \ldots, n, i \neq j .\right.
$$

A neural network describes a population of physically interconnected nerve cells. Communication between cells is mainly due to electrochemical processes. In this article, we focus on analyzing the behavior of a set of neurons connected with a given topology by electrical signals. Thus, we consider a complex system based on a network of interactions between neurons in which each network node is modeled by a PDE of FHN type. The article contains the following sections: (1) introduction; (2) definition of synchronization, especially identical synchronization. Here, we seek sufficient conditions for a certain type of synchronization in our network; (3) investigation of minimal value of coupling strength for synchronization incomplete network to occur and numerical experiments that give an insight into the influence of neurons on minimal coupling strength needed to obtain synchronization in network. Our numerical simulations show that when the number of nodes in the graph grows, the network becomes easier to synchronize; and (4) conclusion.

\section{Identical synchronization of a complete network of $n$ systems of reaction- diffusion on FitzHugh-Nagumo type}

Synchronization is a ubiquitous feature in many natural systems and nonlinear science. The word "synchronization" is of Greek origin, with syn as "common" and chronos as "time", which means having the same behavior at the same time. Therefore, the synchronization of two dynamical systems usually means that one system copies the movement of the other. When the behavior of many systems is synchronized, these systems are called synchronous. Studies by Aziz-Alaoui (2006) and Corson (2009) suggested that a phenomenon of synchronization may appear in a network of many weakly coupled oscillators. A broad variety of applications have emerged to increase the power of lasers, synchronize the output of electric circuits, control oscillations in chemical reactions or encode electronic messages for secure communications. Here are some synchronization regimes (Aziz-Alaoui, 2006):

- Identical (or complete) synchronization, which is defined as the coincidence of states of interacting systems;

- Generalized synchronization, which extends the identical synchronization phenomenon and implies the presence of some functional relation between two coupled systems; if this relationship is the identity, we recover the identical synchronization; 
- Phase synchronization, which means driving of phases of chaotic oscillators, whereas their amplitudes remain uncorrelated;

- Lag synchronization, which appears as a coincidence of shifted-in-time states of two systems.

In this article, we are interested in the identical synchronization (Ambrosio \& AzizAlaoui, 2013) incomplete network which means that each node connects to all other nodes of the network. For example, Figure 2 showed the complete graphs from 2 to 10 nodes and complete graphs of 40 nodes. In this study, each node represents a neuron modeled by a system of reaction-diffusion equations on FHN type and each edge represents a synaptic connection modeled by a coupling function.

Definition 1: Let $S_{i}=\left(u_{i}, v_{i}\right), i=1,2, \ldots, n$ and $S=\left(S_{1}, S_{2}, \ldots, S_{n}\right)$ be a network. We say that $S$ is identical synchronization if,

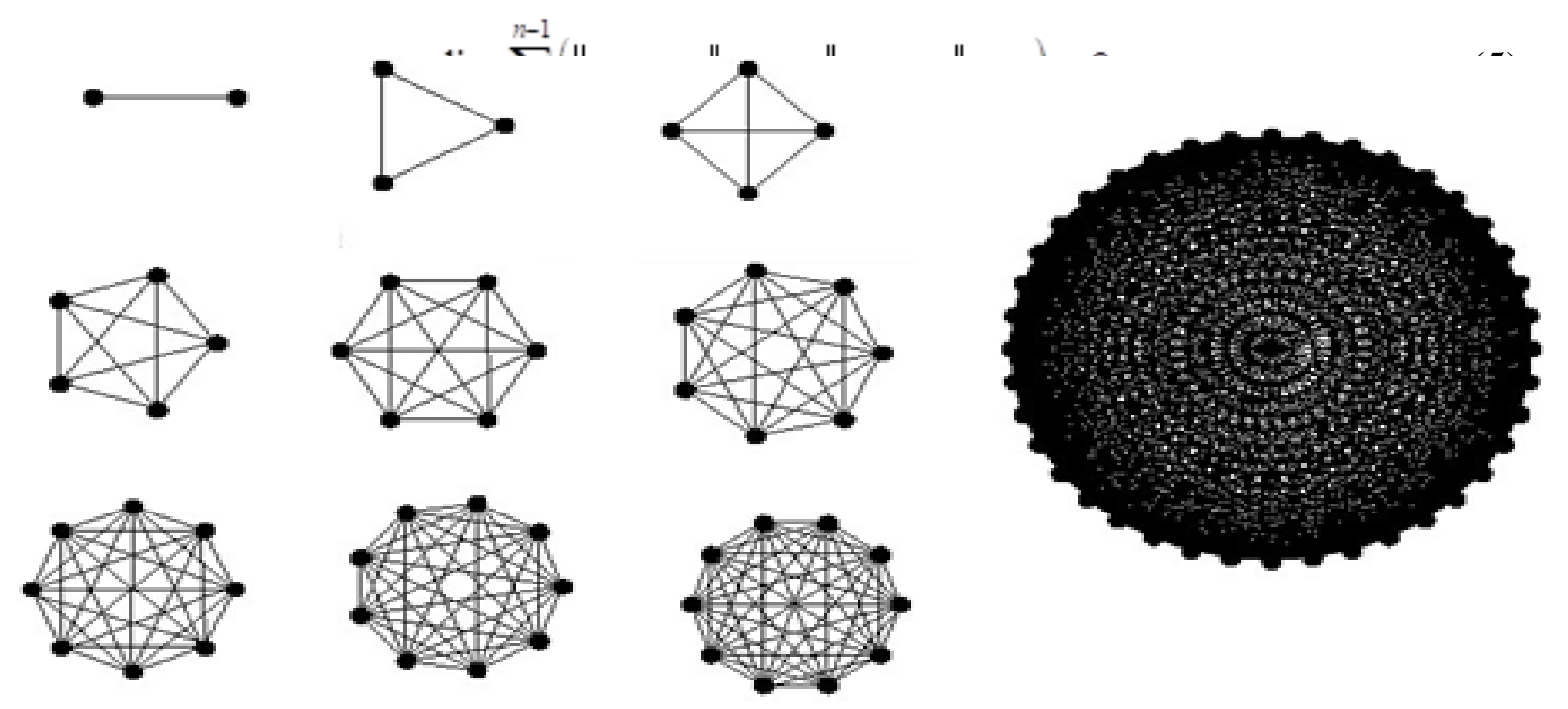

Figure 2. Complete graphs from 2 to 10 nodes and complete graphs of 40 nodes. In our study, each node represents a neuron modeled by a system of reaction-diffusion equations on FHN type and each edge represents a synaptic connection modeled by a coupling function.

We consider a system of $n$ "neurons" (1) bi-directionally coupled with the electrical synapses,

$$
\left\{\begin{array}{l}
\varepsilon u_{i t}=f\left(u_{i}\right)-v_{i}+d_{1 u_{i}} \Delta u_{i}-g_{n} \sum_{j=1, j=i}^{n}\left(u_{i}-u_{j}\right) \quad i=1,2, \ldots, n, \\
v_{i t}=a u_{i}-b v_{i}+c
\end{array}\right.
$$

where $g_{n}$ is the coupling strength between $u_{i}$ and $u_{j}$. 
Theorem 1: Suppose that:

$$
g_{n}>\frac{M}{n} \operatorname{andd}_{u_{i}}=d_{u_{j}}, i, j=1,2, \ldots, n,
$$

where $M=\sup _{u \in B, x \in \mathbb{R}} \sum_{k=1}^{3} \frac{f^{(k)}(u)}{k !} x^{k-1}, B$ is a compact interval including $u$ and $f^{(k)}(u)$ is the $k$ th derivative off with respect tou. Then the network (4) synchronizes in the sense of definition 1.

Proof: Let $\Phi(t)=\frac{1}{2}\left[\sum_{i=2}^{n}\left(a \varepsilon \int_{\Omega}\left(u_{i}-u_{1}\right)^{2} d x+\int_{\Omega}\left(v_{i}-v_{1}\right)^{2} d x\right)\right]$.

By deriving the function $\Phi(t)$, we have the following,

$$
\begin{aligned}
& \frac{d \Phi(t)}{d t}=\sum_{i=2}^{n} \int_{\Omega}\left[a \varepsilon\left(u_{i}-u_{1}\right)\left(u_{\dot{t}}-u_{1 t}\right)+\left(v_{i}-v_{1}\right)\left(v_{\dot{t}}-v_{1 t}\right)\right] d x \\
& =\sum_{i=2}^{n} \int_{\Omega}\left[a\left(u_{i}-u u_{1}\right)\left(f\left(u u_{i}\right)-v_{i}+d_{1 u_{i}} \Delta u_{i}-g_{n} \sum_{k=1, k \neq i}^{n}\left(u u_{i}-u l_{k}\right)-f\left(u u_{1}\right)+v_{1}-d_{1 u_{i}} \Delta u_{1}+g_{n} \sum_{i=2}^{n}\left(u u_{1}-u u_{j}\right)\right)\right. \\
& \left.+\left(v_{i}-v_{i}\right)\left(a\left(u_{i}-u u_{1}\right)-b\left(v_{i}-v_{1}\right)\right)\right] d x \\
& \leq \sum_{i=2}^{n} \int_{\Omega}\left[a\left(u u_{i}-u u_{1}\right)\left(f\left(u u_{i}\right)-f\left(u u_{1}\right)-n g_{n}\left(u u_{i}-u u_{1}\right)\right)-b\left(v_{i}-v_{1}\right)^{2}\right] d x \\
& \leq \sum_{i=2}^{n} \int_{n}\left[a\left(u u_{i}-u u_{1}\right)^{2}\left(f^{\prime}\left(u_{1}\right)-n g_{n}+\sum_{k=2}^{3} \frac{f^{(k)}\left(u_{1}\right)}{k !}\left(u_{i}-u u_{1}\right)^{k-1}\right)-b\left(v_{i}-v_{1}\right)^{2}\right] d x \\
& \leq \sum_{i=2}^{n} \int_{\Omega}\left[a\left(u_{i}-u u_{1}\right)^{2}\left(M-n g_{n}\right)-b\left(v_{i}-v_{1}\right)^{2}\right] d x \\
& \text { If } g_{n}>\frac{M}{n} \text {, we have } \\
& \frac{d \Phi(t)}{d t} \leq-\beta \Phi(t) \Rightarrow \Phi(t) \leq \Phi(0) e^{-\beta t}
\end{aligned}
$$

where $\beta=\min \left(2 \frac{n g_{n}-M}{\varepsilon}, 2 b\right)$. Thus, we have the synchronization if the coupling strength verifies $g_{n}>\frac{M}{n}$.

If $f$ is a cubic, we deduce the following corollary.

Corollary 1: Suppose that $f$ is a cubic function, $f(u)=m_{3} u^{3}+m_{2} u^{2}+m_{1} u+m_{0}$, where $m_{3}, m_{2}, m_{1}, m_{0}$ are constants with $m_{3}<0$ and if,

$$
g_{n}>\frac{1}{n}\left(m_{1}-\frac{m_{2}^{2}}{3 m_{3}}\right)
$$


the network $S=\left(\left(u_{1}, v_{1}\right),\left(u_{2}, v_{2}\right), \ldots,\left(u_{n}, v_{n}\right)\right)$ synchronizes in the sense of definition 1 .

\section{Numerical simulations}

In the following, we present the numerical results obtained by integrating the system (4) where $n=3, f(u)=-u^{3}+3 u$, and with the following parameter values: $a=1, b=0.001, c=0, \varepsilon=0.1, d_{u}=0.05$. The integration of system was realized by using $\mathrm{C}++$, on $[0 ; T] \times \Omega=[0 ; 200] \times[0 ; 100] \times[0 ; 100]$.

Figure 3 illustrates the phenomenon of synchronization. The simulations show that the system synchronizes from the value $g_{3}=0.025$. In the figures (a), (b), (f), (g), (k), (l), (p), (q), we represented the phase portraits $\left(u_{1}\left(x_{1}, x_{2}, t\right), u_{2}\left(x_{1}, x_{2}, t\right)\right)$ and $\left(u_{2}\left(x_{1}, x_{2}, t\right), u_{3}\left(x_{1}, x_{2}, t\right)\right)$ for $t \in[0 ; T]$ and for all $\left(x_{1}, x_{2}\right) \in \Omega$. We observe (figure (p) and (q)) that for $g_{3}=0.025$, $u_{1}\left(x_{1}, x_{2}, t\right) \approx u_{2}\left(x_{1}, x_{2}, t\right)$ and $u_{2}\left(x_{1}, x_{2}, t\right) \approx u_{3}\left(x_{1}, x_{2}, t\right)$ for all $t \in[0 ; T]$ and for all $\left(x_{1}, x_{2}\right) \in \Omega$. In the figures (c), (d), (e), (h), (i), (j), (m), (n), (o), (r), (s), (t), we represented the iso values of $u_{i}\left(x_{1}, x_{2}, 190\right), i=1,2,3$. The results show that $g_{3}=0.025$ the obtained patterns are "identical".

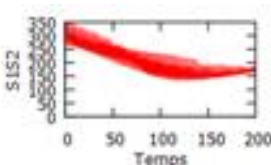

(a)

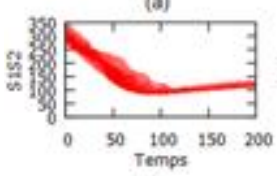

(i)
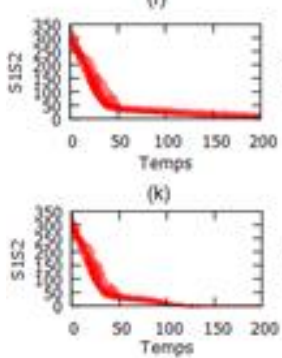

(9)

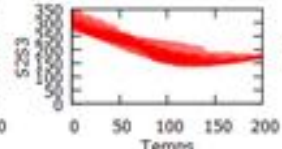

(b)

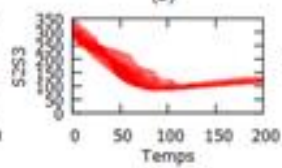

(g)

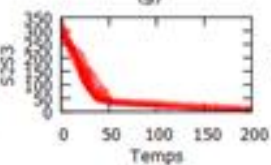

(1)

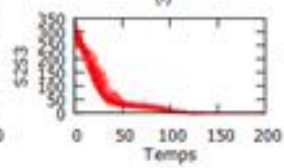

(q)

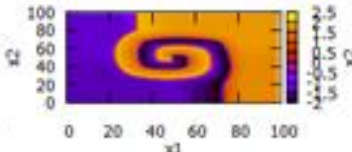

(c)

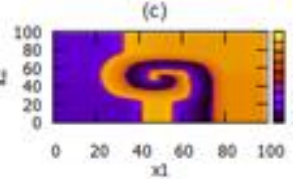

(h)

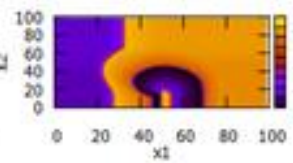

(m)

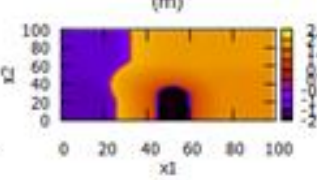

(r)

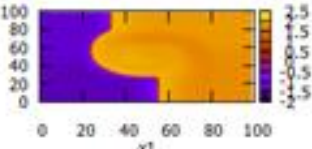

(d)

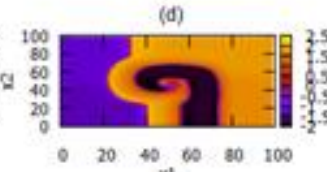

(1)

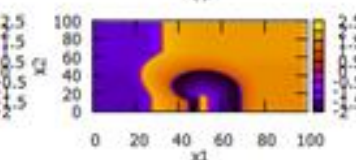

(n)

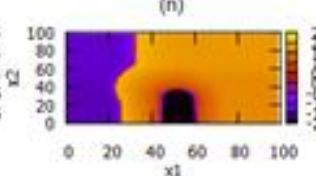

(s)

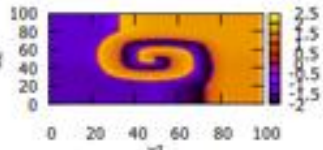

$\begin{array}{llllll}0 & 20 & 40 & 60 & 80 & 100\end{array}$

(e)

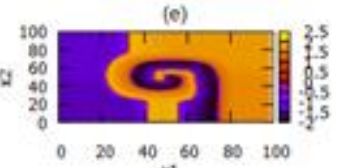

(6)

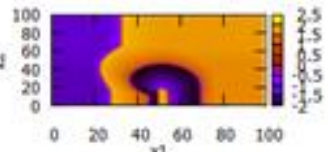

(o)

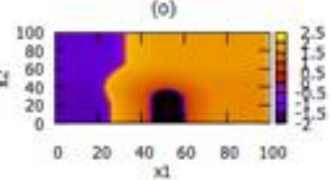

(i)

Figure 3. Synchronization of a complete network of three linearly coupled "neurons" with $f(u)=-u^{3}+3 u, a=1, b=0.001, c=0, \varepsilon=0.1, d_{u}=0.05$. The synchronization occurs for $g_{3}=0.025$.

Before synchronization, $g_{3}=0.005$ figure (a) represents the temporal dynamic of $u_{2}$ with respect to $u_{1}$, for all $\left(x_{1}, x_{2}\right) \in \Omega$; figure (b) represents the temporal dynamic of $u_{3}$ with respect to $u_{2}$; the figure (c) represents the iso values of $u_{1}\left(x_{1}, x_{2}, 190\right)$; similarly the figures (d) and (e)

represent the iso values of $u_{2}\left(x_{1}, x_{2}, 190\right)$ and $u_{3}\left(x_{1}, x_{2}, 190\right)$; the similar simulations are reproduced for $g_{3}=0.01$ (figures (f), (g), (h), (i), (j)), $g_{3}=0.023$ (figures (k), (l), (m), (n), (o)) and $g_{3}=0.005$ (figures (p), (q), (r), (s), (t)). For the value $g_{3}=0.005$, we observe the synchronization of three "neurons" 
We are interested in the minimal values of coupling strength $g_{n}$ to observe a phenomenon of synchronization between $n$ subsystems modeling the function of a neuron. Thus, in the case of three linearly coupled neurons, we note that for the coupling strength over or equal to $g_{3}=0.005$, these neurons have asynchronous behavior (Figure 3). By doing similarly for the complete networks of linearly identical coupled neurons, we obtain the values of coupling strength reported in Table 1.

\section{Table 1}

This table gives the minimal coupling strength necessary to observe a phenomenon of synchronization of $\mathrm{n}$ linearly coupled neurons, with the parameters: $f(u)=-u^{3}+3 u, a=1, b=0.001, c=0, \varepsilon=0.1, d_{u}=0.05$.

\begin{tabular}{|c|c|c|c|c|c|c|c|c|c|}
\hline$n$ & $\mathbf{3}$ & $\mathbf{4}$ & $\mathbf{5}$ & $\mathbf{6}$ & $\mathbf{7}$ & $\mathbf{8}$ & $\mathbf{9}$ & $\mathbf{1 0}$ & $\mathbf{1 1}$ \\
\hline$g_{n}$ & 0.025 & 0.015 & 0.012 & 0.009 & 0.008 & 0.007 & 0.006 & 0.005 & 0.0045 \\
\hline$n$ & 12 & 13 & 14 & 15 & 16 & 17 & 18 & 19 & 20 \\
\hline$g_{n}$ & 0.004 & 0.0038 & 0.0035 & 0.0032 & 0.003 & 0.0028 & 0.0026 & 0.0024 & 0.0023 \\
\hline
\end{tabular}

Source: The researcher's data analysis

Following these numerical experiments, we see that the coupling strength required for observing the synchronization of $n$ neurons depends on the number of neurons. Indeed, the points in Figure 4 represent the coupling strength of synchronization according to the number of neurons incomplete network, and the red curve represents the representative one,

$$
g_{n}=\frac{0.051}{n-1}-0.00041
$$

where $n$ is the number of neurons in the network. Thus, the coupling strength necessary to obtain the synchronization of $n$ neurons follows this law.

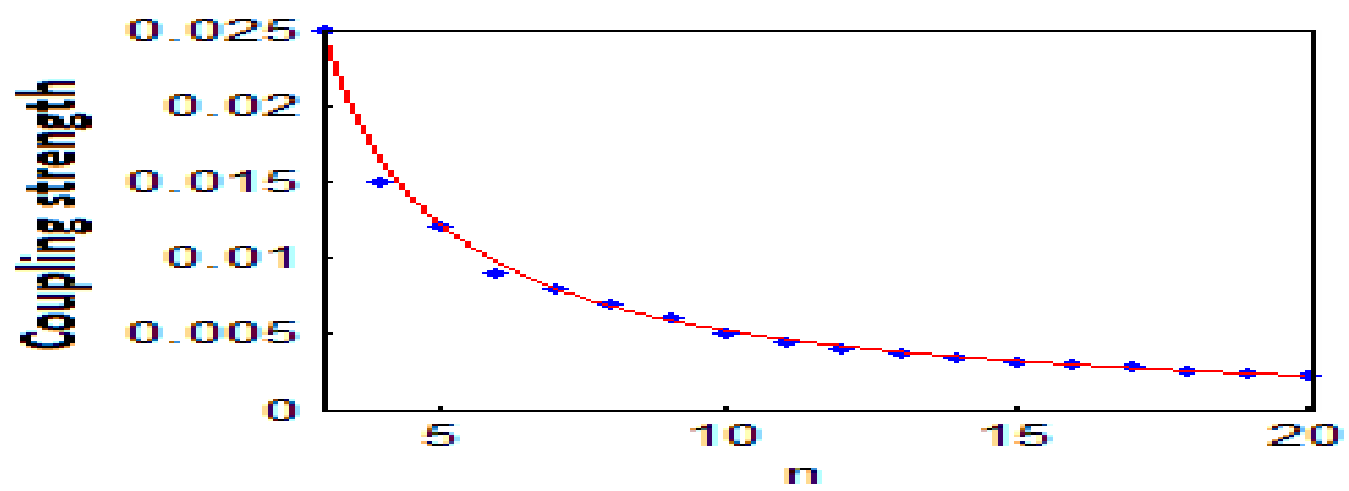

Figure 4. Figure 4 showed the evolution of the coupling strength $g_{n}$ for which the synchronization of $\mathrm{n}$ neurons takes place according to the number $\mathrm{n}$ linearly coupled neurons incomplete network. Thus, this evolution follows the law $g_{n}=\frac{0.051}{n-1}-0.00041$. 


\section{Conclusion}

This study investigated a phenomenon of synchronization incomplete network of $n$ coupled systems of reaction-diffusion on Fitzhugh-Nagumo type. From theorem 1, we got $g_{n}>\frac{M}{n}$ which shows that the bigger the value of $n$, the smaller the $g_{n}$. Numerically, we found that the synchronization is stable when we exceed a certain threshold of coupling strength and depends on the number of "neurons" in graphs. The bigger the number of "neurons" is, the easier the phenomenon of synchronization will be obtained. Then, a compromise between the theoretical and numerical results can be reached. In addition, it is necessary to conduct further studies on the identical synchronization incomplete network coupled with chemical synapse.

\section{References}

Ambrosio, B. (2009). Propagation d'ondes dans un milieu excitable: Simulations numériques et approche analytique. (Master's thesis). University Pierre and Marie Curie-Paris 6, Paris, France.

Ambrosio, B., \& Aziz-Alaoui, M. A. (2012). Synchronisation dans un réseau d'équations aux dérivées partielles de type FitzHugh-Nagumo. In Actes du colloque EDP-Normandie (pp. 119-131).

Ambrosio, B., \& Aziz-Alaoui, M. A. (2012). Synchronization and control of coupled reactiondiffusion systems of the FitzHugh-Nagumo-type. Computers and Mathematics with Applications, 64(5), 934-943.

Ambrosio, B., \& Aziz-Alaoui, M. A. (2013). Synchronization and control of a network of coupled reaction-diffusion systems of generalized FitzHugh-Nagumo type. ESAIM: Proceedings, 39, 15-24.

Aziz-Alaoui, M. A. (2006). Synchronization of chaos. Encyclopedia of Mathematical Physics, Elsevier, 5, 213-226.

Corson, N. (2009). Dynamique d'un modèle neuronal, synchronisation et complexité. Retrieved January $\quad 10, \quad 2017, \quad$ from https://tel.archivesouvertes.fr/file/index/docid/458094/filename/these_NC.pdf

Corson N., \& Aziz-Alaoui, M. A. (2009). Complex emergent properties in synchronized neuronal oscillations. In M. A. Aziz-Alaoui \& C. Bertelle (Eds.), From system complexity to emergent properties (pp. 243-259). Paris, France: Springer.

Ermentrout, G. B., \& Terman, D. H. (2009). Mathematical foundations of neurosciences. Berlin, Germany: Springer.

Hodgkin, A. L., \& Huxley, A. F. (1952). A quantitative description of membrane current and its application to conduction and excitation in nerve. The Journal of Physiology, 117, 500544.

Izhikevich, E. M. (2005). Dynamical systems in neuroscience: The geometry of excitability and bursting. London, England: The MIT Press, Cambrige, Massachusetts. 
Izhikevich, E. M. (2007). Dynamical systems in neuroscience. London, England: The MIT Press.

Keener, J. P., \& Sneyd, J. (2009). Mathematical physiology. New York, NY: Springer.

Murray, J. D. (2010). Mathematical biology. New York, NY: Springer.

Nagumo, J., Arimoto, S., \& Yoshizawa, S. (1962). An active pulse transmission line simulating nerve axon. Proceedings of the IEEE, 50(10), 2061-2070. 